\title{
Loculated pneumothorax with a deep sulcus sign
}

\author{
Daniel Hernandez MD, Rita Medrano MD, David Sotello MD, Andres Yepes MD, Ebtesam Islam MD, PhD
}

\begin{abstract}
A 61-year-old man with amyotrophic lateral sclerosis, s/p tracheostomy, and ventilator dependence was admitted for evaluation of respiratory distress, fever, and increased secretions. Broad spectrum antibiotics were started due to bilateral infiltrates on his admission chest $x$-ray. One day after admission the patient developed severe left shoulder pain and tachycardia. Chest $\mathrm{x}$-ray showed a pneumothorax with a positive deep sulcus sign in the left hemithorax (Figure 1). A 16 French surgical chest tube was placed with the complication of a persistent air-leak (Figure 2). Eventually, the pneumothorax recurred despite presence of the surgical chest tube, and a second pigtail chest tube was inserted by the Interventional Radiology service (Figure 3).
\end{abstract}

\section{Discussion}

Loculated pneumothorax is defined as air trapped inside an air pocket between the pleural layers. ${ }^{1}$ This air does not move and remains localized, unlike the typical pneumothorax in which the air moves to the anterosuperior region of the lung. Loculated pneumothorax is associated with severe inflammatory processes, including the acute respiratory distress syndrome (ARDS). In ARDS the inflammation reaction forms adhesions dividing the ple ural cavity in multiple pockets into which air can leak from inflammation mediated lung injury. ${ }^{2}$

Clinical presentation of loculated pneumothorax can be vague and difficult to suspect, given that patients are usually critically ill and the only positive clinical signs are hypoxemia and tachycardia. Additionally, loculated pneumothoraces do not present with the typical findings on chest $\mathrm{x}$-rays with a well demarcated visceral pleural edge with homogeneous

Corresponding author: David Sotello Contact Information: David.sotello@ttuhsc.edu DOI: 10.12746/swrccc.v6i26.497

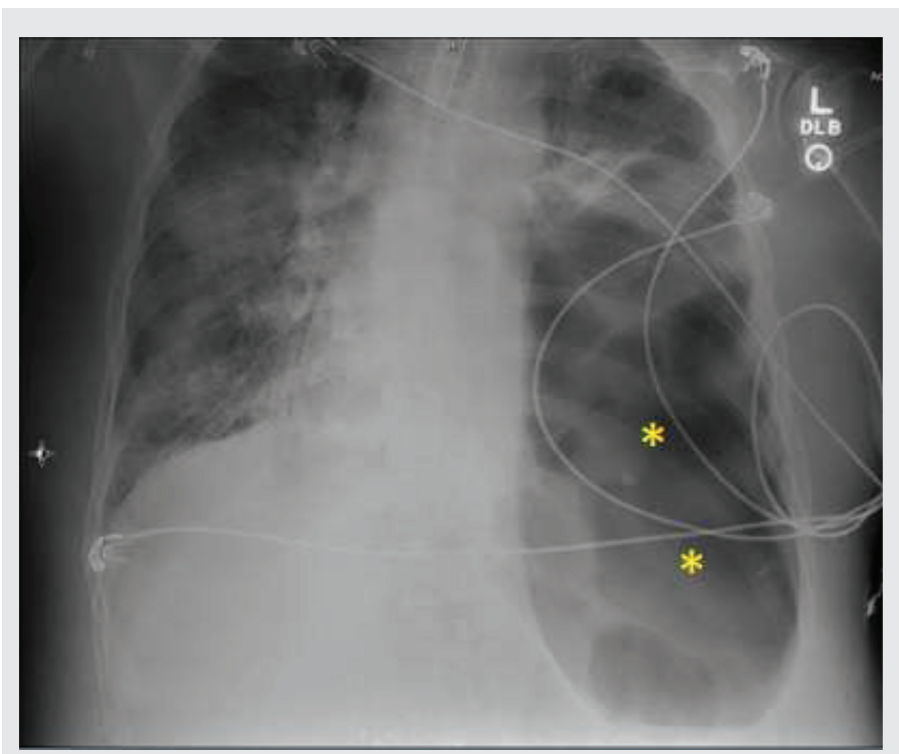

Figure 1. AP chest $x$-ray showing left loculated pneumothorax $(*)$ with positive deep sulcus sign. Note the concave left costophrenic angle.

distribution along the thoracic cavity depending on the pneumothorax extent. Clinicians should look for air in the pleural cavity which usually looks like an abnormal lucency with enhancement of surrounding organs.

As seen in our patient in Figure 1, a deep sulcus sign is characterized by a lucency seen at the costophrenic border with deepening of the angle; this is a common indirect finding on supine chest $x$-ray for pneumothorax. ${ }^{3}$ However, chest $x$-rays are unreliable in detecting small air collections as they underestimate the amount of air in the pleural cavity. If there is any suspicion about the diagnosis, chest computed tomography (CT) is the gold standard to identify pneumothorax. Ultrasound has also shown high sensitivity and specificity when compared to chest CT as it can identify small collections of air and determine their extension by tracking the sliding lung sign, which is a visible sliding of the parietal pleura over the visceral pleura. ${ }^{4}$ 


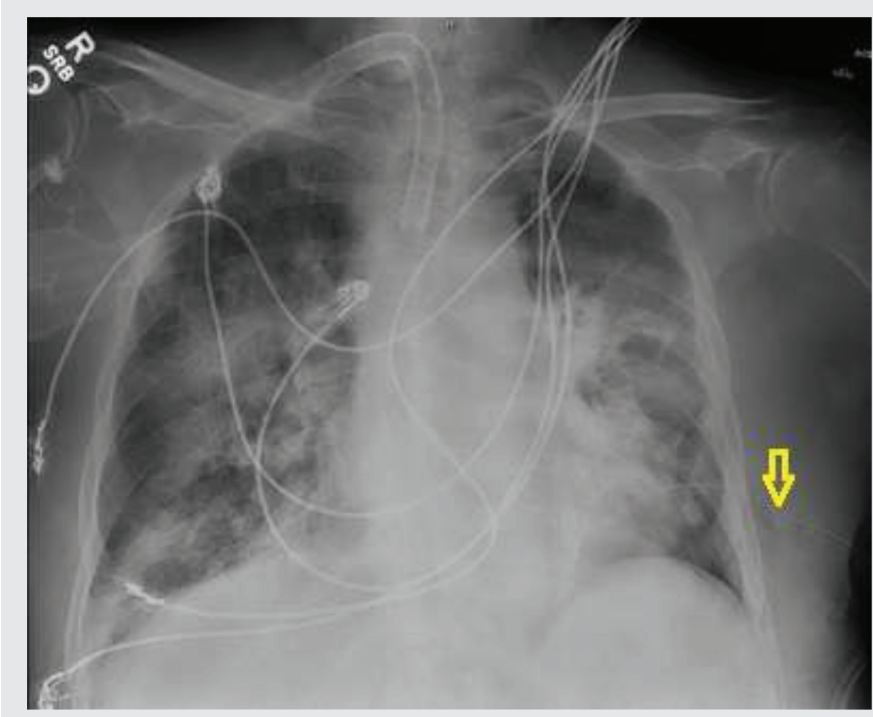

Pneumothorax management is usually with chest tube placement ipsilateral to the location of the air. However, a loculated pneumothorax requires direct drainage using imaging guidance to locate the pocket. Imaging techniques used to guide pneumothorax drainage include fluoroscopy, chest CT, and chest sonogram. Chest CT and ultrasonography are preferred modalities as described previously. Fluoroscopy was used in the past but has been discontinued due to the availability of newer and more effective techniques. ${ }^{4}$

Keywords: pneumothorax, deep sulcus sign, chest tube

Figure 2. AP chest $x$-ray showing interval resolution of loculated pneumothorax after a 16 French thoracostomy tube placement (arrow). Note the convexity of left costophrenic angle.

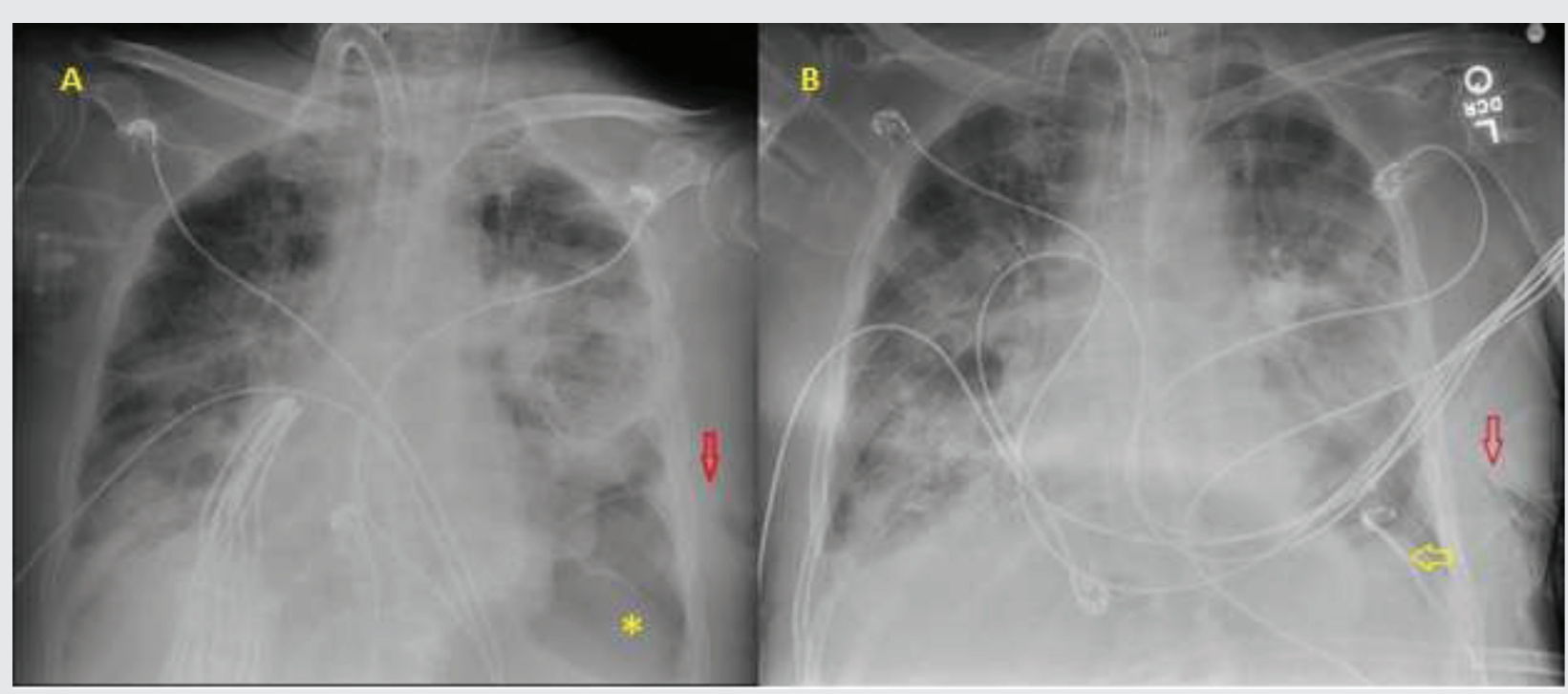

Figure 3. Panel A shows an AP chest $x$-ray showing interval recurrence of left pneumothorax $(*)$ despite 16 French thoracostomy tube (red arrow). Panel B shows interval placement of 8 French pigtail thoracostomy tube (yellow arrow). 
From: The Department of Internal Medicine at Texas Tech University Health Sciences Center in Lubbock, Texas and Universidad Dr. Jose Matias Delgado, El Salvador (DH).

Submitted: 9/4/2018

Accepted: 9/10/2018

Reviewer: Eman Attaya MD

Conflicts of interest: none

This work is licensed under a Creative Commons Attribution-ShareAlike 4.0 International License

\section{REFERENCES}

1. Boland G, Lee M, Sutcliffe N, et al. Loculated pneumothoraces in patients with acute respiratory disease treated with mechanical ventilation: preliminary observations after imageguided drainage. J Vasc Interv Radiol 1996;7(2):247-252.

2. Chon K, vanSonnenberg E, D'Agostino H, et al. CT-guided catheter drainage of loculated thoracic air collections in mechanically ventilated patients with acute respiratory distress syndrome. AJR Am J Roentgenol 1999;173(5):1345-1350.

3. Gordon R. The deep sulcus sign. Radiology 1980;136(1): 25-27.

4. Paramasivan E, Bodenham A. Air leaks, pneumothorax and chest drains. Continuing Education in Anesthesia Critical Care Pain 2008;8(6):204-209. 